\title{
Sustained Release of a Monoclonal Antibody from Electrochemically Prepared Mesoporous Silicon Oxide
}

\author{
Dr. Jennifer S. Andrew, \\ Department of Chemistry and Biochemistry, University of California, San Diego, 9500, Gilman Dr, \\ La Jolla, CA 92093 (USA) \\ Dr. Emily J. Anglin, \\ Department of Chemistry and Biochemistry, University of California, San Diego, 9500, Gilman Dr, \\ La Jolla, CA 92093 (USA) \\ Elizabeth C. Wu, \\ Bioengineering Department, University of California, San Diego, CA 92093-0412 (USA) \\ Michelle Y. Chen, \\ Bioengineering Department, University of California, San Diego, CA 92093-0412 (USA) \\ Dr. Lingyun Cheng, \\ Jacobs Retina Center at Shiley Eye Center, University of California, San Diego, San Diego, CA \\ 92092 (USA) \\ Dr. William R. Freeman, and \\ Jacobs Retina Center at Shiley Eye Center, University of California, San Diego, San Diego, CA \\ 92092 (USA) \\ Prof. Michael J. Sailor ${ }^{\star}$ \\ Department of Chemistry and Biochemistry, University of California, San Diego, 9500, Gilman Dr, \\ La Jolla, CA 92093 (USA)
}

\section{Abstract}

Nanostructured mesoporous silica $\left(\mathrm{SiO}_{2}\right)$ films are used to load and release the monoclonal antibody bevacizumab (Avastin) in vitro. A biocompatible and biodegradable form of mesoporous $\mathrm{SiO}_{2}$ is prepared by electrochemical etching of single crystalline $\mathrm{Si}$, followed by thermal oxidation in air at $800{ }^{\circ} \mathrm{C}$. Porous $\mathrm{SiO}_{2}$ exhibits a negative surface charge at physiological $\mathrm{pH}(7.4)$, allowing it to spontaneously adsorb the positively charged antibody from an aqueous phosphate buffered saline solution. This electrostatic adsorption allows bevacizumab to be concentrated by $>100 \times\left(300 \mathrm{mg}\right.$ bevacziumab per gram of porous $\mathrm{SiO}_{2}$ when loaded from a $1 \mathrm{mg} \mathrm{mL}^{-1}$ solution of bevacziumab). Drug loading is monitored by optical interferometric measurements of the thin porous film. A two-component Bruggeman effective medium model is employed to calculate percent porosity and film thickness, and is further used to determine the extent of drug loading into the porous $\mathrm{SiO}_{2}$ film. In vitro drug release profiles are characterized by an enzyme-linked immunosorbent assay (ELISA), which confirms that the antibody is released in its active, VEGFbinding form. The nanostructured delivery system described here provides a sustained release of the monoclonal antibody where approximately $98 \%$ of drug is released over a period of one month. 


\section{Introduction}

Current pharmaceutical technology relies mainly on the systemic delivery of drugs.

However, for drugs with limited therapeutic windows or where biological barriers must be crossed, systemic delivery is incapable of providing sufficient quantities of drug at the target tissues. Higher doses increase risk of unwanted side effects or toxicity. There is, therefore, an important and unmet need for drug delivery systems that provide minimally invasive, controllable drug release. Additionally, orally administered drugs are susceptible to degradation when exposed to the harsh conditions present in the body, specifically in the gastrointestinal tract. The development of drug delivery vehicles that can temporarily protect a therapeutic, deliver it to specific tissues, and then release it in a controlled fashion has been pursued to improve the efficacy of pharmaceutical therapies. Previous work has demonstrated the utility of the approach, using liposomes,[1] microemulsions,[2] polymeric spheres,[3] or various nano- and micro-particles and devices[4,5] as host materials. Whereas nanoscale drug delivery vehicles are generally developed with an intravenous injection protocol in mind, particles larger than $\sim 1 \mu \mathrm{m}$ do not circulate in the bloodstream well and they are better suited for local administration. One such area of interest is the eye.

Delivering therapeutics to the eye is a particularly challenging task, due to the difficulty of crossing the blood-retinal barrier. Systemic administrations of drugs for ocular diseases require large and potentially toxic doses to deliver only a small fraction of drug at the target. [6] In light of this, intravitreal injection has become the standard method for administering drugs to the eye. However, the short half-life of drugs in the vitreous requires frequent injections of high doses of drug. For example, the anti-angiogenic drug bevacizumab has a half-life in the vitreous of only 3-4.3 days.[7,8] Each injection introduces risk of infection or hemorrhage, which can lead to permanent vision loss.[9] We reasoned that these limitations may be overcome by loading into a porous $\mathrm{SiO}_{2}$ host, providing sustained release over longer periods of time. This would be particularly advantageous in the treatment of agerelated macular degeneration (ARMD), which is the primary cause of blindness in the developed world.[10] Previously, we demonstrated that micron-scale particles derived from porous $\mathrm{SiO}_{2}$ can be delivered to rabbit eyes via intravitreal injection. The particles showed no evidence of toxicity for periods of $>4$ months in vivo[11] and they degraded completely to soluble and excretable orthosilicates. We have also shown that the particles can be prepared as photonic crystals such that they display characteristic color changes as they degrade, which might provide the clinician with a colorimetric indicator to predict the course of a therapy.[12] Previous work has shown the ability of porous $\mathrm{SiO}_{2}$ to incorporate a range of biomolecules, including bovine serum albumin,[13,14] active enzymes,[15] immunoglobulins, [16,17] and protein A,[16] although the loading and release of a therapeutically relevant antibody from such materials, in a therapeutically relevant timescale, has not been demonstrated.

A significant amount of work on porous Si as a drug delivery material has been published, beginning with the initial discoveries of Canham and coworkers in the mid 1990s;[18-24] in particular the biodegradability and biocompatibility advantages of such a nanostructured host have been demonstrated.[25,26] Additionally, nanostructured porous Si has a reactive surface that can be chemically modified for enhanced drug loading $[27,28]$ or to control degradation rate, $[25,26]$ and its surface area-to-volume ratio, pore size, and open porosity can be readily tuned by adjusting the electrochemical preparation conditions.[29] This ability to design in a predetermined degradation profile is highly desired in order for the formulator to respond to changing treatment protocols or to adjust the therapy to suit a particular patient. 
Under physiological conditions porous Si degrades into orthosilicic acid, the natural form of silicon in the body which is readily removed by the kidneys.[20,30] The in vitro degradation pathway for porous $\mathrm{Si}$ involves two steps: oxidation to $\mathrm{SiO}_{2}$ followed by hydrolysis to soluble orthosilicic acid species. Although water is an effective oxidant for the first step, the reducing power of porous $\mathrm{Si}$ can lead to undesirable side reactions-for example, the common MTT cellular viability assay will give a false positive result due to reduction of the active diphenyltetrazolium indicator by Si or Si hydride species.[31] Therefore, the suitability of porous $\mathrm{Si}$ as a host for drugs that are easily reduced is questionable. This issue is ameliorated by pre-oxidizing the porous $\mathrm{Si}$ material to porous $\mathrm{SiO}_{2 .}[31]$

This study aimed to develop a porous $\mathrm{SiO}_{2}$ host that can effectively load and sustainably release the monoclonal antibody bevacizumab (Avastin) in its active form. Bevacizumab (trade name Avastin) is one of a growing number of monoclonal antibody-based drugs. $[32,33]$ Bevacizumab targets angiogenesis pathways by binding with vascular endothelial growth factor (VEGF).[34] In the treatment of colon cancer, bevacizumab has been shown to be effective in combination with traditional chemotherapy. This is attributed to the fact that bevacizumab inhibits tumor angiogenesis, working to renormalize tumor vasculature to allow more effective delivery of the chemotherapeutic. Bevacizumab has also proven to be effective in the treatment of certain ocular diseases, specifically in treating choridal neovascularization associated with wet age-related macular degeneration (ARMD).[35] In contrast to some cancers, ARMD cannot be cured, and the anti-angiogenic drug must be maintained at a minimal therapeutic concentration in the patient indefinitely in order to keep the disease at bay. Current treatment requires that extremely high concentrations of bevacizumab be injected intravitreally, in order for the drug to remain above the therapeutic limit for a reasonable amount of time.

In this paper, we demonstrate the capabilities of electrochemically prepared porous $\mathrm{SiO}_{2}$ for long-term sustained release of a monoclonal antibody-based drug. We focus on porous films still attached to the Si substrate to allow more accurate quantification of drug loading and release characteristics. To our knowledge, this is the first demonstration of this material's ability to release a functional antibody-based therapeutic for extended periods.

\section{Results and Discussion}

\subsection{Preparation of Porous Silica}

Highly doped $\mathrm{p}^{++}$type Si wafers were etched at a current density of $233 \mathrm{~mA} \mathrm{~cm}^{-2}$, for 1 min, generating a mesoporous Si film $15 \mu \mathrm{m}$ thick. The surface of freshly etched porous Si is hydride terminated $(\mathrm{Si}-\mathrm{H})$, which is highly reactive and dissolves readily in aqueous solution.[36,37] In this study the mesoporous Si samples were thermally oxidized at $800{ }^{\circ} \mathrm{C}$ for $1 \mathrm{~h}$, yielding near-complete conversion to silicon dioxide, or silica $\left(\mathrm{SiO}_{2}\right)$. The average pore size of the samples is $25 \pm 5 \mathrm{~nm}$ (Figure 1). This pore size was chosen because it allowed for incorporation of bevacizumab $(150 \mathrm{kDa})$, which has a hydrodynamic diameter of $11 \mathrm{~nm}$.

The porosity and thickness of the films were determined using the spectroscopic liquid infiltration method (SLIM).[38] Briefly, the optical thickness (OT), $2 n L$, of the porous silica film can be determined from the interferometric reflectance spectrum, where $n$ is the index of refraction and $L$ is the film thickness. The porous silica films used in this study are optically flat and transparent, and their reflectivity spectra contain Fabry-Pérot interference fringes.[39] The position of the fringe maxima and minima correspond to constructive and destructive interference at the air/porous $\mathrm{SiO}_{2}$ (or liquid/porous $\mathrm{SiO}_{2}$ ) and porous $\mathrm{SiO}_{2} /$ bulk Si interfaces, given by the relationship $m \lambda=2 n L$, where $m$ is the spectral order of a fringe at a given wavelength, $\lambda$. The value of $2 n L$, the effective optical thickness, is readily obtained 
from the Fourier transform of the reflectivity spectrum (Figure 2). When the media within the pores is changed, the average index of refraction of the porous layer changes, causing a shift in optical thickness. For example, when buffer infiltrates the pores, the value of $n$ increases, due to the fact that buffer has a larger index of refraction than air, resulting in an increase in the measured optical thickness of the film.

To determine film thickness and open porosity, the index of refraction obtained from the optical thickness measurement is modeled using the two-component Bruggeman effective medium approximation (EMA). Two measurements, one of the dry film (in air) and the other with the sample immersed in phosphate buffered saline (PBS) solution are obtained, and simultaneous solution of the two resulting EMA equations yields unique values for both the porosity and the thickness of the film. The refractive indices of $\mathrm{SiO}_{2}$, air, and PBS were assumed to be $1.455,1$, and 1.336 , respectively. The calculated value of film thickness for all samples in this study is $15.0 \pm 0.7 \mu \mathrm{m}$ and the open porosity is $81 \% \pm 2 \%$. The thickness measurements obtained are in good agreement with those obtained from electron microscopy. Separate gravimetric measurements[38] verify these results, agreeing within $5 \%$ of the values for porosity calculated using the Bruggeman equation.

\subsection{Bevacizumab Loading}

Porous $\mathrm{SiO}_{2}$ prepared by the above method is reasonably stable in aqueous media, is hydrophilic, and it displays a negative surface charge, allowing for effective loading of a positively charged drug from aqueous solution. The optical technique described above can be extended to quantify protein infiltration and adsorption within the porous $\mathrm{SiO}_{2}$ film. Most proteins possess refractive indices larger than aqueous buffers, such as the PBS used here. Therefore, protein adsorption onto the porous $\mathrm{SiO}_{2}$ surface will result in an increase in the measured optical thickness, $2 n L$. Assuming the thickness of the porous film remains constant, the increase in optical thickness can be attributed to an increase in the effective index of refraction. A three-component Bruggeman effective medium model is applied to determine the amount of protein loaded within the pores, where the three components are $\mathrm{SiO}_{2}$, the buffer solution, and the immobilized protein (bevacizumab). Drug loading was performed using a flow cell fitted with an optical window in order to monitor the drug loading process in real time. This monitoring method is well established for biosensor applications.[14,16,40] Here, we extend the technique to monitor the rate and extent of bevacizumab loading. A typical loading experiment is shown in Figure 3. In each experiment, a baseline was established by flowing PBS solution through the flow cell for 15 min prior to bevacizumab introduction. Once the drug was introduced, a rapid increase in the optical thickness was observed. The bevacizumab solution was flowed through the cell in a closed loop for $6 \mathrm{~h}$, at which point the optical thickness attained a steady-state value. After loading, the films were rinsed with PBS for 15 min to remove any bevacizumab that was not strongly adsorbed onto the inner pore walls. During this rinsing step, the optical thickness decreased quickly to a second steady-state value, indicating that the remaining bevacizumab is strongly adsorbed in the porous matrix.

The effect of concentration of the bevacizumab loading solution on loading efficiency was investigated for concentration values of $0.125,0.5$, and $1.0 \mathrm{mg} \mathrm{mL}^{-1}$. The amount of adsorbed bevacizumab increases with the concentration of free drug in the loading solution (Figure 4). After free drug had been removed with a pure buffer rinse, the amount of immobilized drug was quantified using the method described above (Table 1). To quantify the extent of drug immobilization in the porous $\mathrm{SiO}_{2}$ matrix, a concentration factor, $C F$, was calculated. The value of $C F$ is defined as the ratio of the mass of the drug in the porous $\mathrm{SiO}_{2}$ film per unit volume to the mass of drug in the loading solution per unit volume, Equation 1: 


$$
C F=\frac{[\text { bevacizumab }]_{\text {pores }}}{[\text { bevacizumab }]_{\text {solution }}}
$$

The concentration factors (Table 1) reveal that the antibody s concentrated in the porous $\mathrm{SiO}_{2}$ film relative to the free solution. The affinity of bevacizumab for the porous $\mathrm{SiO}_{2}$ surface is attributed to strong electrostatic interactions (Scheme 1). Scheme 1 depicts bevacizumab loading throughout the pore volume, which is verified by optical interferometry. If loading occurred solely at the top of this pore, this would be manifest in the reflectivity spectrum as the appearance of a double layer.[41] Silica has an isoelectric point (pI) of $\sim 2,[42]$ whereas the $\mathrm{pI}$ of bevaizumab is 7.6,[43] therefore under the drug loading conditions ( $\mathrm{pH} 7.4$ ), the negatively charged silica surface will attract the positively charged bevacizumab. To verify that bevacizumab binds electrostatically to the porous $\mathrm{SiO}_{2}$ matrix, the loading experiment was repeated in buffer solutions with $\mathrm{pH} 4.7,5.3$, and 7.5 (Figure 5). Due to the instability of porous $\mathrm{SiO}_{2}$ in basic conditions we were unable to use $\mathrm{pH}$ values > the $\mathrm{pI}$ (7.6) of bevacizumab. However, at the lower $\mathrm{pH}$ values of 4.7 and 5.3 the net positive charge on bevacizumab is larger, and it therefore is expected to interact more strongly with the negatively charged porous $\mathrm{SiO}_{2}$ matrix, resulting in the increased loading observed. Although electrostatic forces are likely the dominant mechanism for bevacizumab loading, it is also possible that localized dissolution and re-precipitation of silicic acid during drug loading could physically trap the drug within the porous $\mathrm{SiO}_{2}$ matrix.[44]

The quantity of bevacizumab loaded was verified by measurement of bevacizumab concentration in the loading solution before and after loading, using an enzyme-linked immunosorbent assay (ELISA). The optical monitoring technique slightly underestimates the amount of bevacizumab loaded relative to the ELISA result. For example, the sample shown in Figure 3 exhibits a percent increase in optical thickness of $0.3 \%$, corresponding to a loading of approximately $150 \mu \mathrm{g}$ of protein in the porous $\mathrm{SiO}_{2}$ film. However, the ELISA assay reveals a loading of $\sim 250 \mu \mathrm{g}$. The discrepancy is attributed to partial dissolution of the porous $\mathrm{SiO}_{2}$ film during the loading experiments, which results in a slight blue shift of the optically measured baseline (Figure 3, "PBS-control") that was not accounted for in the optical measurements.

\subsection{Bevacizumab Release}

Bevacizumab release experiments were performed in a flow cell with a chamber volume of $4.5 \mathrm{~mL}$, simulating the volume of the vitreous compartment of the human eye.[45] The flow rate of buffer through the chamber was chosen such that the dissolved contents would exhibit a half-life of 3.5 days in the chamber. This flow rate was selected in order to resemble the half-life of free bevacizumab in the human vitreous.[7,8] The effluent from the flow chamber was collected approximately every $24 \mathrm{~h}$ for 30 days, and the amount of bevacizumab was quantified using an ELISA assay. Control experiments confirmed that dissolved silica did not interfere with the ELISA binding assay. Because the ELISA assay used in this paper is a VEGF binding assay, it only measures bevacizumab that retains its functionality, or its ability to bind to VEGF. The release profile is shown in Figure 6, and exhibits sustained release over a period of $>30$ days, at which point $~ 94 \%$ of the loaded bevacizumab has been released.

In order to assess the relevance of the drug-loaded materials for treatment of humans with neovascular ARMD (wet form ARMD), it is necessary to assess the concentration of drug released with respect to therapeutically relevant concentrations. Figure 7 shows the amount of free bevacizumab in the release chamber as a function of time, revealing the concentration of drug that would be available in the vitreous of a human patient. The lowest 
effective therapeutic concentration $\left(22 \mathrm{ng} \mathrm{mL}^{-1}\right)$ [46] and the upper toxic dose (1.3 $\mathrm{mg}$ $\mathrm{mL}^{-1}$ )[47] are also shown in Figure 6. A toxic limit for bevacizumab has not been reported in the literature. In clinical practice, Avastin is typically administered in doses of $1.25 \mathrm{mg}$ per $4.5 \mathrm{~mL}$, but it has been injected at doses of up to $2.5 \mathrm{mg}$ per $4.5 \mathrm{~mL}$ with no toxicity.[48] However, toxicity of ranbizumab (Lucentis) has been reported for doses of $2 \mathrm{mg}$ per $4.5 \mathrm{~mL}$. Ranbizumab is comprised of only the antigen binding, or Fab, fragment of an anti-VEGF antibody. For the purpose of this study, the toxic dose for bevacizumab was extrapolated by calculating the amount of bevacizumab that would produce the same concentration of the Fab fragment; this concentration corresponds to $1.3 \mathrm{mg} \mathrm{mL}^{-1}$. A theoretical trace depicting the drug concentration if it were to be injected into the cell at day 0 as free drug is also shown (Figure 6, "free drug (calc.)"). For comparison purposes, the amount of drug used in the calculation of the "free drug" case is equal to the quantity of drug that was contained in the porous $\mathrm{SiO}_{2}$ film at time 0 . Figure 6 illustrates the effect of sequestering the monoclonal antibody into the porous, bioresorbable carrier: the initial concentration of drug to which the tissues would be exposed is lowered by a factor of 10 , while still providing a therapeutically effective dose for the entire 4 week treatment period. It should be pointed out that the total amount of drug used in these simulated treatment experiments is 5-fold less than a typical clinical dose $(1.25 \mathrm{mg}$ per $4.5 \mathrm{~mL})$ used to treat ARMD. In the typical clinical dose $0.05 \mathrm{~mL}$ of a $25 \mathrm{mg} \mathrm{mL}{ }^{-1}$ stock bevacizumab is injected for a dose of $1.25 \mathrm{mg}$, by loading $130 \mathrm{mg}$ bevacizumab per $\mathrm{mL}$ of porous silica we have effectively increased the concentration by a factor of $\sim 5$.

Previous work has shown that porous $\mathrm{SiO}_{2}$ microparticles can be injected into rabbit eyes with no observable toxicity, with a residence time of $>4$ months.[11] In subsequent experiments, we have found the residence time of these particles in the rabbit eye can be extended up to 8 months, during which period the particles are observed to dissolve completely. The data presented here indicate that a microparticle formulation of porous $\mathrm{SiO}_{2}$ containing the same net quantity of bevacizumab that is administered as the free drug in the clinical dose could provide a therapeutic level of drug in the eye for $>4$ months. This represents a significant improvement over the current clinical regimen of one injection every 4 weeks.[49]

\section{Conclusions}

A monoclonal antibody-based drug, bevacizumab (Avastin), can be loaded and released from nanostructured porous $\mathrm{SiO}_{2}$ films. The negative surface charge of the porous $\mathrm{SiO}_{2}$ matrix allows the positively charged antibody to be concentrated within the porous matrix by a factor of up to 470 , relative to the concentration of free drug in aqueous solution. The maximum loading efficiency for this electrostatic adsorption method is $\sim 300 \mathrm{mg}$ of antibody per gram of porous $\mathrm{SiO}_{2}$. By monitoring the Fabry-Pérot interference fringes in the optical reflectivity spectra of the porous $\mathrm{SiO}_{2}$ films, the extent of drug loading can be monitored non-destructively and in real-time. Drug release was quantified using an ELISA to verify that the released drug retained its functional antigen binding properties. The porous $\mathrm{SiO}_{2}$ matrix releases bevacizumab over a period of $>30$ days, with no initial burst observed in the release profile.

\section{Experimental Section}

\section{Materials}

Highly doped p-type single crystalline silicon wafers ( $\mathrm{p}++,<100\rangle$ orientation) with a resistivity of $0.75 \mathrm{~m} \Omega \mathrm{cm}$ were purchased from Siltronix, inc. Avastin (bevacizumab) was obtained from Genentech, Inc. VEGF and bovine serum albumin (BSA, $\geq 98 \%$ ELISA grade) were obtained from Sigma Aldrich Chemical Co. Horseradish peroxidase conjugated 
goat anti-human immunoglubulin (anti-IgG-HRP) was obtained from Millipore. PBS (1×, pH 7.4) was obtained from Gibco, Inc. Dulbecco's phosphate buffered saline (DPBS, $1 \times$ ) was obtained from Mediatech, Inc. Tris buffered saline (TBS, 1×, pH 7.4), SuperSignal ELISA Pico Chemiluminescent substrate, aqueous hydrofluoric acid (HF, 48\%), and ethanol $(99.9 \%)$ were obtained from Fisher Scientific.

\section{Porous Silica Preparation}

Porous Si samples were prepared by anodization of highly doped p++ Si wafers in an ethanolic hydrofluoric acid solution (3:1 v/v 48\% aqueous HF:ethanol) using a two electrode configuration, using a platinum mesh cathode. Si wafers with an exposed area of $8.6 \mathrm{~cm}^{2}$ were placed onto aluminum foil, to create a back contact to the Si anode, and loaded into a Teflon etch cell. A constant current density of $233 \mathrm{~mA} \mathrm{~cm}{ }^{-2}$ was applied for $60 \mathrm{~s}$. After etching, the samples were thoroughly rinsed with ethanol and dried under a stream of nitrogen. The freshly etched films were thermally oxidized at $800{ }^{\circ} \mathrm{C}$ for $1 \mathrm{~h}$. After oxidation, the samples were allowed to cool to room temperature.

\section{Bevacizumab Loading}

Drug loading into the porous $\mathrm{SiO}_{2}$ films was carried out using a flow cell apparatus using a flow rate of $1 \mathrm{~mL} \mathrm{~min}^{-1}$ for $6 \mathrm{~h}$ in a closed loop configuration. Briefly, the flow cell was constructed of plexiglass, consisting of an inlet and an outlet, and was connected to a peristaltic pump. The flow cell, as previously described,[14] was fitted with an optical window to allow acquisition of reflectivity spectra from the porous $\mathrm{SiO}_{2}$ surface during the drug loading experiments. Drug loading solutions $\left(0.125-1 \mathrm{mg} \mathrm{mL}^{-1}\right)$ were prepared by diluting bevacizumab in PBS. After loading, the films were rinsed with PBS for 15 min to remove any weakly adsorbed drug.

Demonstration of electrostatic adsorption mechanism experiments were also carried out using the flow cell apparatus, as described above, and drug was flowed in a closed loop configuration for $1 \mathrm{~h}$. Drug loading solutions were prepared by diluting bevacizumab in $\mathrm{pH}$ buffer solutions ( $\mathrm{pH} 4.7,5.3,7.5)$ at a concentration of $1 \mathrm{mg} \mathrm{mL}^{-1}$. After loading, the films were rinsed with the appropriate buffer to remove any weakly adsorbed drug.

\section{Porous Silica Characterization}

Scanning electron microscopy (SEM, Phillips XL30) was used to determine the pore diameter and film thickness of the porous $\mathrm{SiO}_{2}$ films. Adobe Photoshop CS3 was used to quantify the pore diameter and film thickness from the SEM images, 100 or 15 measurements were made per sample for pore diameter and film thickness, respectively.

Optical reflectivity measurements were also performed to determine the open porosity, film thickness, and amount of drug loading in the porous $\mathrm{SiO}_{2}$ films, as described previously.[38] Briefly, reflectivity data were recorded for wavelengths between 400-1000 nm, with an acquisition time of $100 \mathrm{~ms}$. A discrete fast Fourier transform (FFT) from the Wavemetrics IGOR program library was applied, where the peak of the FFT spectrum yields the film's optical thickness, $2 n L$, where $n$ and $L$ are the index of refraction and thickness of the porous layer, respectively. Using this optical thickness value along with a two component Bruggeman effective medium model, the open porosity and film thickness were calculated, and by extending to a three-component Bruggeman effective medium model the amount drug loaded was determined. The protein possesses a refractive index that is larger than aqueous PBS buffer, and thus protein adsorption generates an increase in the observed value of the effective optical thickness, $2 n L$, of the film. The difference between the reflectance spectrum in buffer and the reflectance spectrum of the film after drug loading is attributed to the presence of adsorbed drug within the pores. The refractive indices for air, becazimab, 
$\mathrm{SiO}_{2}$, and PBS used in the model are $1,1.40,1.445$, and 1.336 , respectively. The analysis provides a volume fraction filling, and to convert this to mass loading the densities of bevacizumab and porous $\mathrm{SiO}_{2}$ are needed, which are taken as $1.4 \mathrm{~g} \mathrm{~cm}^{-3}$ and $2.2 \mathrm{~g} \mathrm{~cm}^{-3}$, respectively. Separate gravimetric measurements were performed on all samples to determine porosity and thickness, and agreed within $10 \%$ of the optical measurements.

\section{Bevacizumab Release}

Drug release experiments were performed using a flow chamber with a chamber volume of $4.5 \mathrm{~mL}$. The bevacizumab-loaded porous $\mathrm{SiO}_{2}$ films were placed in the flow chamber and PBS was flowed through at a rate of $27 \mathrm{~mL} \mathrm{~h}^{-1}$, corresponding to a half-life of 3.5 days for the solution within the chamber. The effluent was collected every $24 \mathrm{~h}$ for 30 days, and the amount of bevacizumab was quantified using an ELISA.

\section{ELISA Assay}

Released bevacizumab concentrations were measured using an ELISA. The detection range of this assay is $0.3125-20 \mathrm{ng} \mathrm{mL}^{-1}$. Human VEGF was immobilized on a Microlite 2 (Fisher Scientific) well plate. As-received VEGF was diluted to $1 \mu \mathrm{g} \mathrm{mL} \mathrm{m}^{-1}$ using DPBS, this solution $(25 \mu \mathrm{L})$ was pipetted into each well, and the well plate was incubated overnight at 4 ${ }^{\circ} \mathrm{C}$. After incubation, the plates were washed five times with $1 \times$ PBS $(200 \mu \mathrm{L})$ and subsequently blocked for $4 \mathrm{~h}$ at $4{ }^{\circ} \mathrm{C}$ with $1 \% \mathrm{BSA}$ in tris-buffered saline (TBS) solution. After blocking, the plates were washed five times with 1X PBS $(200 \mu \mathrm{L})$. For each plate a standard was run, with bevacizumab concentrations ranging from $0.3125-20 \mathrm{ng} \mathrm{mL}^{-1}$. All dilutions were performed using $1 \%$ BSA in TBS and were pipetted (50 $\mu \mathrm{L}$ per well) into the well plates, and then incubated in the dark at room temperature for $1 \mathrm{~h}$. After incubation, the plates were washed five times with 1X PBS $(200 \mu \mathrm{L})$. The amount of bound bevacizumab was detected using a Fc specific goat anti-human immunoglobulin $\mathrm{G}(\mathrm{IgG})$ antibody conjugated with horseradish peroxidase (HRP). This secondary antibody (HRP conjugated anti-human $\mathrm{IgG}$ ) was diluted 1000 times in $1 \%$ BSA in TBS, and $25 \mu \mathrm{L}$ was pipetted into each well and allowed to incubate in the dark for $1 \mathrm{~h}$ at room temperature, followed by washing with 1X PBS $(200 \mu \mathrm{L})$. Quantification of active bevacizumab was achieved by measurement of the intensity of the chemiluminescence signal generated by action of bound HRP on Supersignal ELISA Pico Chemiluminescent substrate. Chemiluminescence was measured using a Molecular Devices Gemini XPS plate reader.

\section{Acknowledgments}

This project was supported by the U.S. National Science Foundation (Grant \# DMR-0806859), and the Jacobs Retina Center (EYO 7366 and EYO 18589). MJS is a member of the Moores UCSD Cancer Center and the UCSD NanoTUMOR Center under which this research was conducted and partially supported by NIH grant U54 CA 119335. The authors gratefully acknowledge Dr. Frederique Cunin, Dr. Daniel Brunel, Dr. Daniel Lerner, and Dr. Sylvie Begu of the CNRS labs at the Institut Charles Gerhardt, Montpellier, France, Dr. Claudia Pacholski, Dr. Michael P. Schwartz, Dr. Sandy Chou, and Dr. Karsten Hartvigsen for helpful discussions.

\section{References}

1. Sengupta S, Eavarone D, Capila I, Zhao G, Watson N, Kiziltepe T, Sasisekharan R. Nature 2005;436:568. [PubMed: 16049491]

2. Spernath A, Aserin A. Adv Colloid Interface Sci 2006;128:47. [PubMed: 17229398]

3. Uhrich KE, Cannizzaro SM, Langer RS, Shakesheff KM. Chem Rev 1999;11:3181. [PubMed: 11749514]

4. Santini JT, Cima MJ, Langer R. Nature 1999;397:335. [PubMed: 9988626]

5. LaVan DA, McGuire T, Langer R. Nat Biotechnol 2003;21:1184. [PubMed: 14520404]

6. Barar J, Javadzadeh AR, Omidi Y. Expert Opin Drug Delivery 2008;5:567. 
7. Bakri SJ, Snyder MR, Reid JM, Pulido JS, Singh RJ. Ophthalmology 2007;114:855. [PubMed: 17467524]

8. Beer PM, Wong SJ, Hammad AM, Falk NS, O’Malley MR, Khan S. Retina 2006;26:871. [PubMed: 17031285]

9. D’Amico DJ, Bird AC. Invest Opthalmol Vis Sci 2004;45:U924.

10. Booth BA, Denham LV, Bouhanik S, Jacob JT, Hill JM. Drug Aging 2007;24:581.

11. Cheng L, Anglin E, Cunin F, Kim D, Sailor MJ, Falkenstein I, Tammewar A, Freeman WR. Br J Ophthalmol 2008;92:705. [PubMed: 18441177]

12. Anglin EJ, Cheng L, Freeman WR, Sailor MJ. Adv Drug Deliv Rev 2008;60:1266. [PubMed: 18508154]

13. Collins BE, Dancil KP, Abbi G, Sailor MJ. Adv Funct Mater 2002;12:187.

14. Dancil KPS, Greiner DP, Sailor MJ. J Am Chem Soc 1999;121:7925.

15. Thomas JC, Pacholski C, Sailor MJ. Lab Chip 2006;6:782. [PubMed: 16738731]

16. Schwartz MP, Alvarez SD, Sailor MJ. Anal Chem 2007;79:327. [PubMed: 17194157]

17. Schwartz MP, Yu C, Alvarez SD, Migliori B, Godin D, Chao L, Sailor MJ. Phys Status Solidi A 2007;204:1444.

18. Canham LT. Adv Mater 1995;7:1033.

19. Canham LT, Newey JP, Reeves CL, Houlton MR, Loni A, Simmons AJ, Cox TI. Adv Mater 1996;8:847.

20. Canham LT, Reeves CL, King DO, Branfield PJ, Crabb JG, Ward MCL. Adv Mater 1996;8:850.

21. Canham LT, Reeves CL, Loni A, Houlton MR, Newey JP, Simons AJ, Cox TI. Thin Solid Films 1997;297:304.

22. Canham LT, Reeves CL, Newey JP, Houlton MR, Cox TI, Buriak JM, Stewart MP. Adv Mater 1999;11:1505.

23. Li X, Coffer JL, Chen YD, Pinizzotto RF, Newey J, Canham LT. J Am Chem Soc 1998;120:11706.

24. Li X, John JS, Coffer JL, Chen Y, Pinizzotto RF, Newey J, Reeves C, Canham LT. Biomed Microdev 2000;2:265.

25. Coffer JL, Montchamp JL, Aimone JB, Weis RP. Phys Status Solidi A 2003;197:336.

26. Salonen J, Kaukonen AM, Hirvonen J, Lehto VP. J Pharm Sci 2008;97:632. [PubMed: 17546667]

27. Buriak JM. Adv Mater 1999;11:265.

28. Wu EC, Park JH, Park J, Segal E, Cunin F, Sailor MJ. ACS Nano 2008;2:2401. [PubMed: 19206408]

29. Zhang XG. J Electrochem Soc 2004;151:C69.

30. Evered, D.; O’Connor, M. Silicon Biochemistry. Wiley; Chichester; New York: 1986.

31. Laaksonen T, Santos H, Vihola H, Salonen J, Riikonen J, Heikkila T, Peltonen L, Kumar N, Murzin DY, Lehto VP, Hirvonen J. Chem Res Toxicol 2007;20:1913. [PubMed: 17990852]

32. Carter P. Nat Rev Cancer 2001;1:118. [PubMed: 11905803]

33. Stern M, Herrmann R. Crit Rev Oncol/Hematol 2005;54:11.

34. Ferrara N, Hillan KJ, Gerber HP, Novotny W. Nature Rev 2004;3:391.

35. Ip MS, Scott IU, Brown GC, Brown MM, Ho AC, Huang SS, Recchia FM. Ophthalmology 2008;115:1837. [PubMed: 18929163]

36. Gupta P, Dillon AC, Bracker AS, George SM. Surf Sci 1991;245:360.

37. Canaria CA, Huang M, Cho Y, Heinrich JL, Lee LI, Shane MJ, Smith RC, Sailor MJ, Miskelly GM. Adv Funct Mater 2002;12:495.

38. Segal E, Perelman LA, Cunin F, Renzo FD, Devoisselle JM, Li YY, Sailor MJ. Adv Funct Mater 2007; 17:1153.

39. Hecht, E. Optics. Addison-Wesley; Reading, MA: 1998.

40. Alvarez SD, Li CP, Chiang CE, Schuller IK, Sailor MJ. ACS Nano 2009;3:3301. [PubMed: 19719156] 
41. Pacholski C, Sartor M, Sailor MJ, Cunin F, Miskelly GM. J Am Chem Soc 2005;127:11636.

[PubMed: 16104739]

42. Parks GA. Chem Rev 1965;65:177.

43. Wiig H, Gyenge CC, Tenstad O. J Physiol-London 2005;567:557. [PubMed: 15994186]

44. Angelos S, Liong M, Choi E, Zink JI. Chem Eng J 2008;137:4.

45. Teichmann KD. J Cataract Refract Surg 2002;28:1886. [PubMed: 12388051]

46. Wang Y, Fei D, Vanderlaan M, Song A. Angiogenesis 2004;7:335. [PubMed: 15886877]

47. Rosenfeld PJ, Schwartz SD, Blumenfranz MS, Miller JW, Haller JA, Reimann JD, Greene WL, Shams N. Opthalmology 2005;112:1048.

48. Bressler NM. Opthalmology 2009;116:S15.

49. Kook D, Wolf A, Kreutzer T, Neubauer A, Strauss R, Ulbig M, Kampik A, Haritoglou C. Retin- J Retin Vitr Dis 2008;28:1053. 

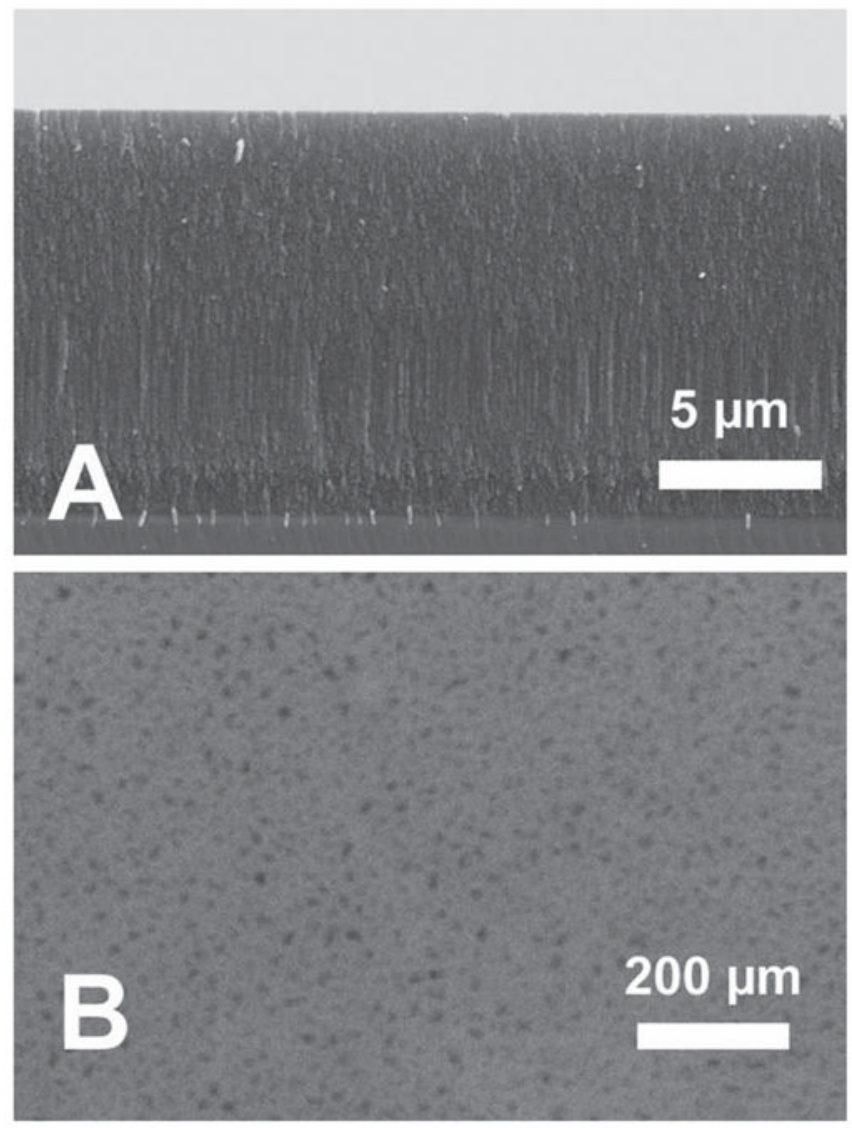

Figure 1.

Scanning electron microscope (secondary electron) images of a porous $\mathrm{SiO}_{2}$ film representative of the samples used in this study. A) Cross-sectional view, the pores are aligned in the $\langle 100\rangle$ direction, and the thickness of the film is $\sim 15 \mu \mathrm{m}$, which agrees within $3 \%$ of the thickness determined by optical interferometry. B) Top (plan) view of the film. Average pore diameter is $\sim 25 \mathrm{~nm}$. 
A)

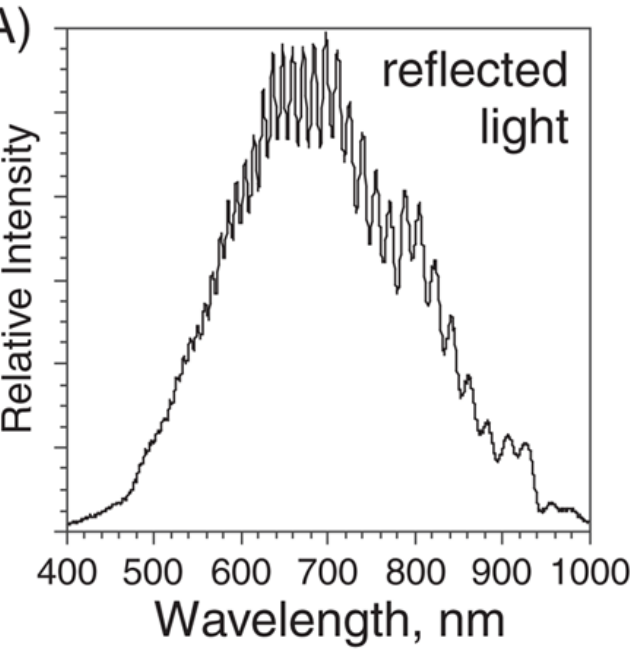

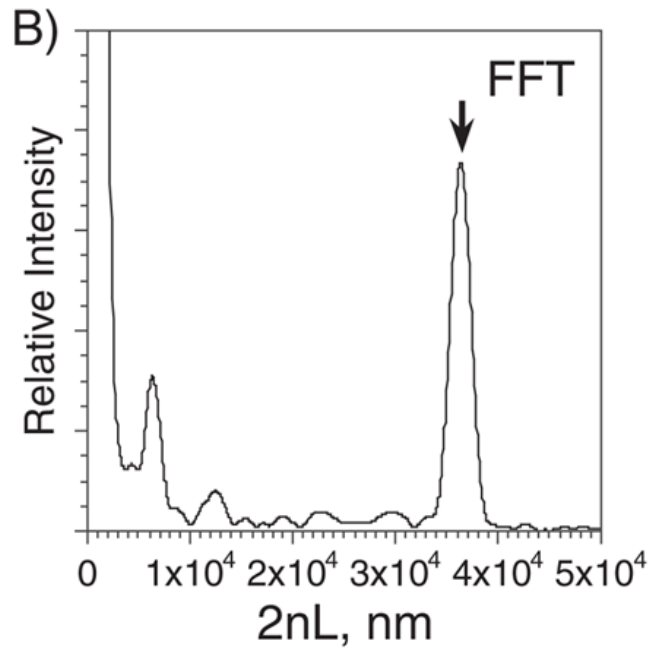

Figure 2.

A) Reflected light spectrum and B) corresponding fast Fourier transform (FFT) spectrum of porous $\mathrm{SiO}_{2}$ film in PBS solution. 


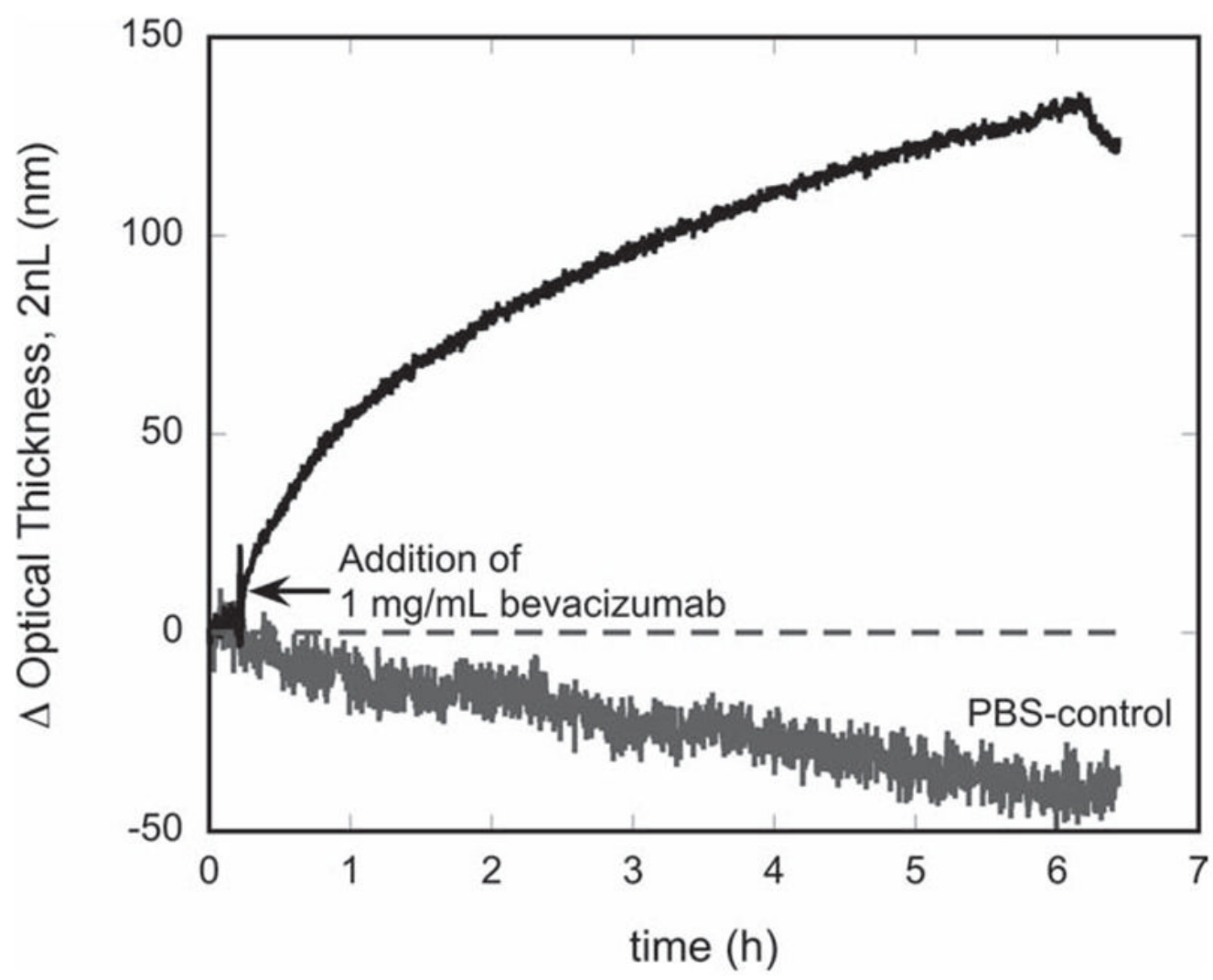

Figure 3.

Drug loading vs time curve for adsorption of bevacizumab in a porous $\mathrm{SiO}_{2}$ film. The film was immersed in aqueous $\mathrm{pH} 7.4$ buffer at time 0 . The top trace represents the change in optical thickness of the porous film after introduction of bevacizumab $\left(1 \mathrm{mg} \mathrm{mL}^{-1}\right)$ to the solution. The bottom trace represents the control, showing the decrease in optical thickness of the porous $\mathrm{SiO}_{2}$ sample during exposure to pure buffer. Buffer is PBS, continuously cycled in a closed loop through the flow cell. The change in optical thickness $(2 n L)$ shown at $t=6 \mathrm{~h}$ represents a total mass loading of $236 \mathrm{mg}$ of bevacizumab per $\mathrm{g}$ of porous $\mathrm{SiO}_{2}(0.13$ $\mathrm{mg}$ bevacizumab per $\mathrm{cm}^{2}$ of porous $\mathrm{SiO}_{2}$ ). 


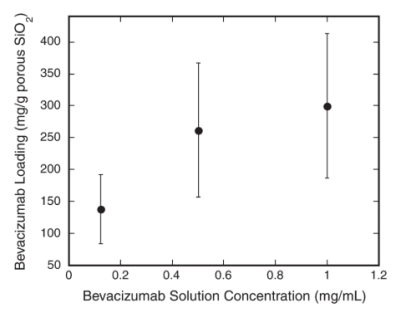

Figure 4.

Relationship between the free solution concentration and amount adsorbed into the porous $\mathrm{SiO}_{2}$ host, for the antibody-based drug bevacizumab. Data are presented in terms of initial concentration of bevacizumab in the aqueous buffer solution into which the porous $\mathrm{SiO}_{2}$ sample was introduced, and the final amount of bevacizumab loaded per gram of porous $\mathrm{SiO}_{2}$. The final amount of bevacizumab loaded was determined after briefly rinsing the free drug from the porous matrix using PBS ( $\mathrm{pH} 7.4)$. 


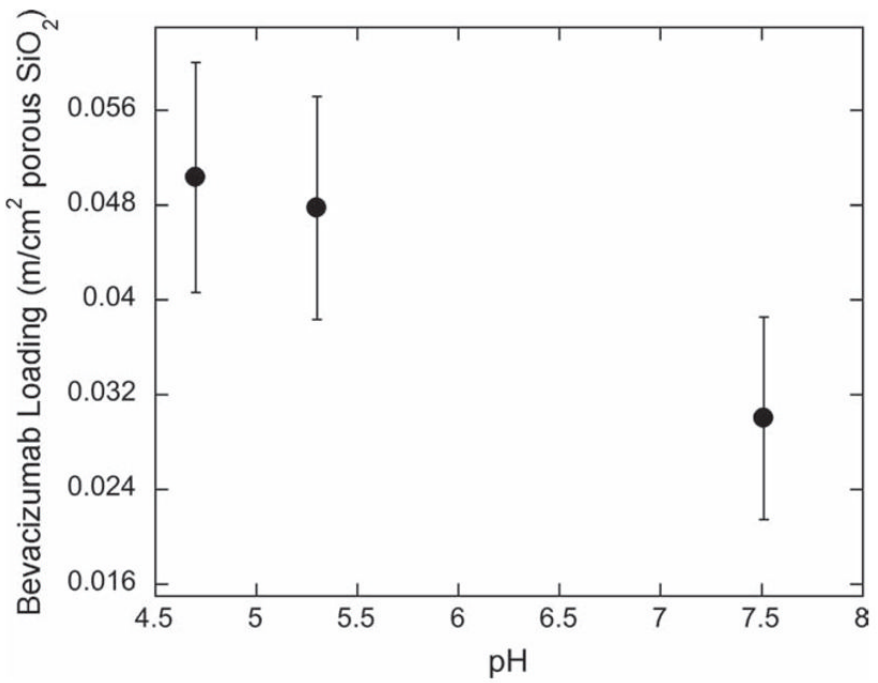

Figure 5.

Relationship between the amount of bevacizumab adsorbed onto the porous $\mathrm{SiO}_{2}$ surface and the $\mathrm{pH}$ of the loading solution. At lower $\mathrm{pH}$ bevacizumab is more positively charged, and therefore interacts more strongly with the negatively charge porous $\mathrm{SiO}_{2}$ surface. As the $\mathrm{pH}$ nears the isoelectric point of bevacizmab this interaction is weakened and less drug is loaded. 


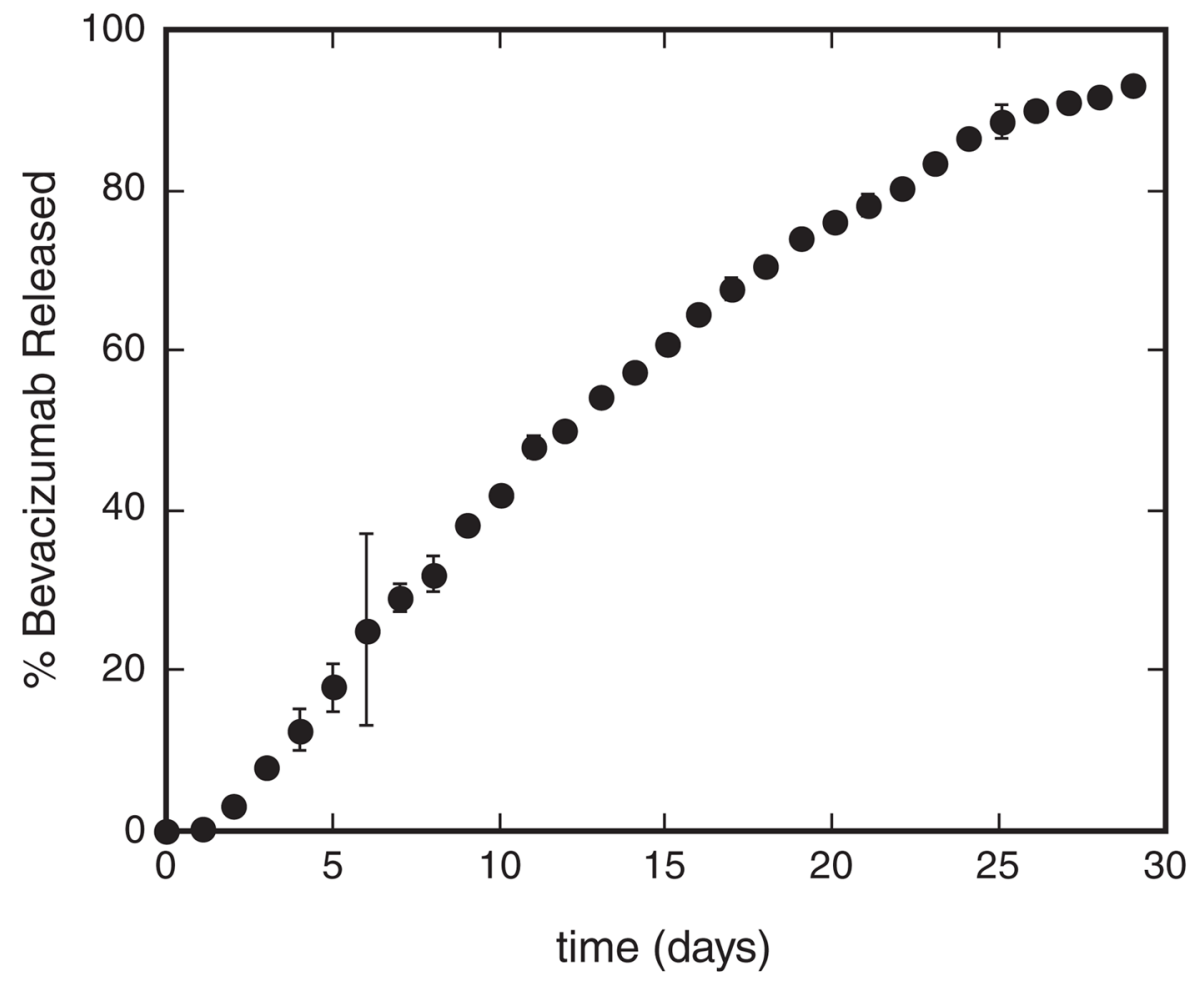

Figure 6.

Release profile of active bevacizumab from porous $\mathrm{SiO}_{2}$ films. The cumulative release of bevacizumab in the effluent as a function of time was quantified using an ELISA assay. Approximately $94 \%$ of the drug is released after 30 days. Total drug loading is $\sim 250 \mu \mathrm{g}$ as verified by optical interferometry measurements as well as by ELISA assay on the loading solution, before and after drug loading. Samples were assayed in triplicate and error bars correspond to the standard deviation. 


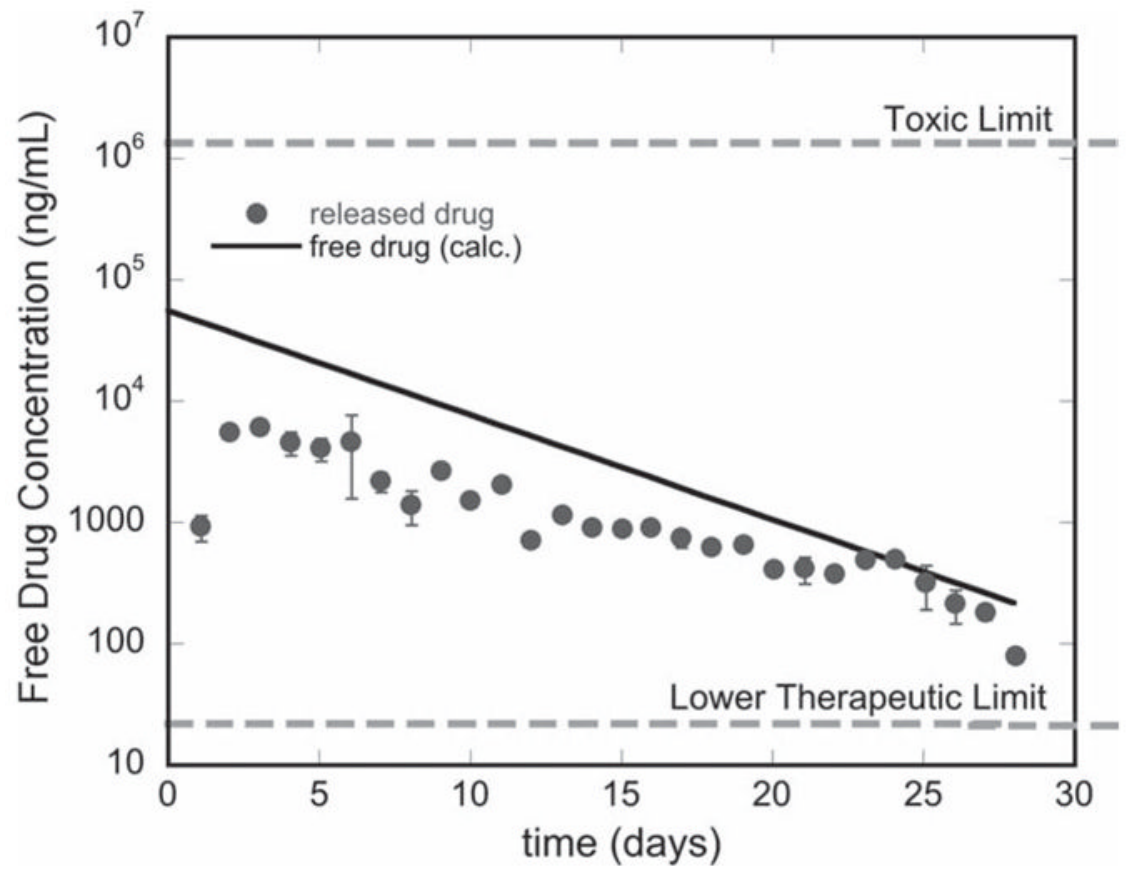

Figure 7.

Concentration of free bevacizumab in the sample chamber during 30 days of continuous buffer flow. At the end of 30 days, $94 \%$ of the drug has been released. The dashed lines indicate the lower and upper therapeutic doses for bevacizumab, indicating that the drug concentration has been maintained within the therapeutic window during the 30 day experiment. Samples were assayed in triplicate and error bars correspond to the standard deviation. Trace labeled free drug (calc.) represents the calculated concentration of bevacizumab if it were to be injected into the cell at day 0 as free drug (rather than sequestered in the porous $\mathrm{SiO}_{2}$ film), and is based on the rate of fresh solution flow into the test chamber. Note that sequestering the drug inside the particles reduces the free drug concentration in the early phase of the experiment by $10 \times$. 


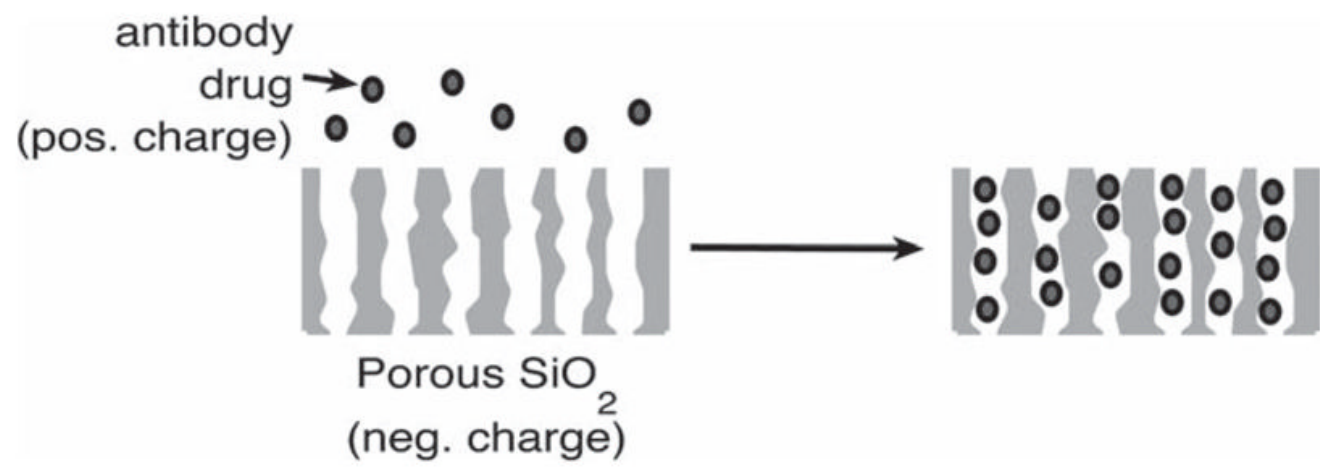

Scheme 1.

Loading of bevacizumab by electrostatic adsorption. Binding to the porous host concentrates the antibody by a factor of $>400$. 
Table 1

Steady-State Bevacizumab Loading Data for Porous $\mathrm{SiO}_{2}$ Films. ${ }^{[a]}$

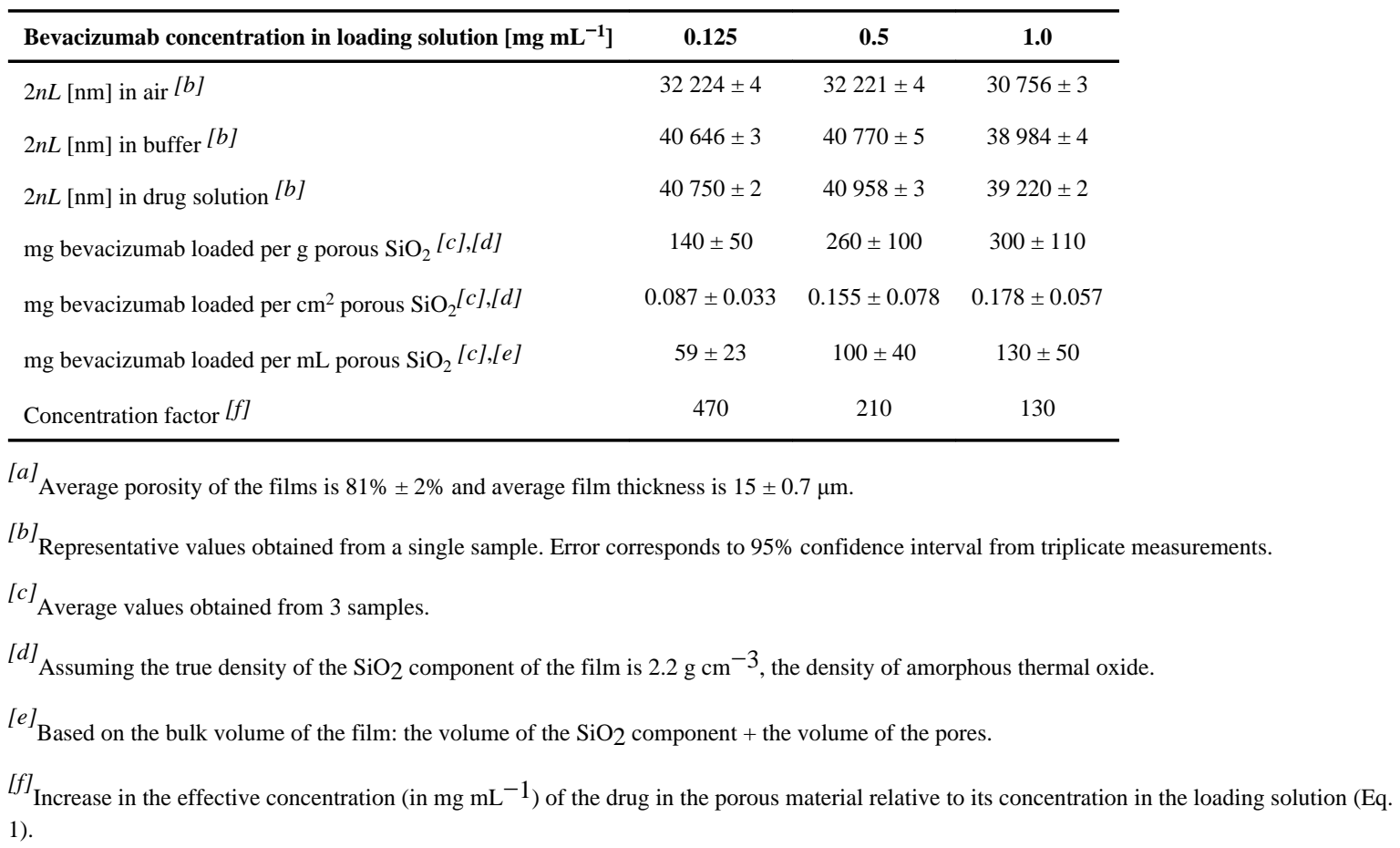

